Canadian

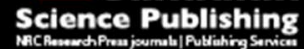

Canadian Journal of Chemistry Revue canadienne de chimie

\title{
Solvent-free preparation of amido-alkyl-phenols catalyzed by trityl chloride under neutral media
}

\begin{tabular}{|r|l|}
\hline Journal: & Canadian Journal of Chemistry \\
\hline Manuscript ID & cjc-2015-0230.R1 \\
\hline Manuscript Type: & Article \\
\hline Date Submitted by the Author: & $03-J u n-2015$ \\
\hline Complete List of Authors: & $\begin{array}{l}\text { Zolfigol, Mohammad Ali; Bu-Ali Sina University, Chemistry } \\
\text { Moosavi-Zare, Ahmad Reza; Bu-Ali Sina University, Chemistry } \\
\text { Derakhshan-Panah, Fateme; Bu-Ali Sina University, Hamedan } \\
\text { 6517838683, Iran, chemistry } \\
\text { Daraei, Masoume; Bu-Ali Sina University, Hamedan 6517838683, Iran, } \\
\text { chemistry }\end{array}$ \\
\hline Keyword: & $\begin{array}{l}\text { amido-alkyl-phenol, multi-component reaction, trityl chloride, neutral, } \\
\text { phenol }\end{array}$ \\
\hline
\end{tabular}




\title{
Solvent-free preparation of amido-alkyl-phenols catalyzed by trityl chloride under neutral media
}

\author{
Ahmad Reza Moosavi-Zare, Mohammad Ali Zolfigol, Fateme Derakhshan-Panah, \\ Masoume Daraei
}

Ahmad Reza Moosavi-Zare. Department of Chemistry, University of Sayyed Jamaleddin Asadabadi, Asadabad, 6541835583, Iran.

Mohammad Ali Zolfigol, Fateme Derakhshan-Panah, Masoume Daraei. Faculty of Chemistry, Bu-Ali Sina University, Hamedan 6517838683, Iran.

${ }^{1}$ Corresponding author (Fax: $\quad+98$ 81323237451; Tel: 9881323237450; E-mail:

moosavizare@yahoo.com). ${ }^{2}$ Corresponding author (Fax: +98 8138257407. E-mail addresses: mzolfigol@yahoo.com). 


\begin{abstract}
One-pot, multi-component condensation reaction of phenols, aromatic aldehydes, and amides in the presence of catalytic amount of trityl chloride $(\mathrm{TrCl})$ as a homogeneous organocatalyst under solvent-free condition to prepare amido-alkyl-phenols has been reported. It is interesting that $\mathrm{TrCl}$ by in situ generation of trityl carbocation with inherent instability catalyzes the reaction.
\end{abstract}

Keywords: amido-alkyl-phenol, multi-component reaction, trityl chloride, neutral, phenol. 


\section{Graphical Abstract}

\section{Solvent-free preparation of amido-alkyl-phenols catalyzed by trityl chloride under neutral media}

Ahmad Reza Moosavi-Zare,* Mohammad Ali Zolfigol,* Fateme Derakhshan-Panah, Masoume Daraei

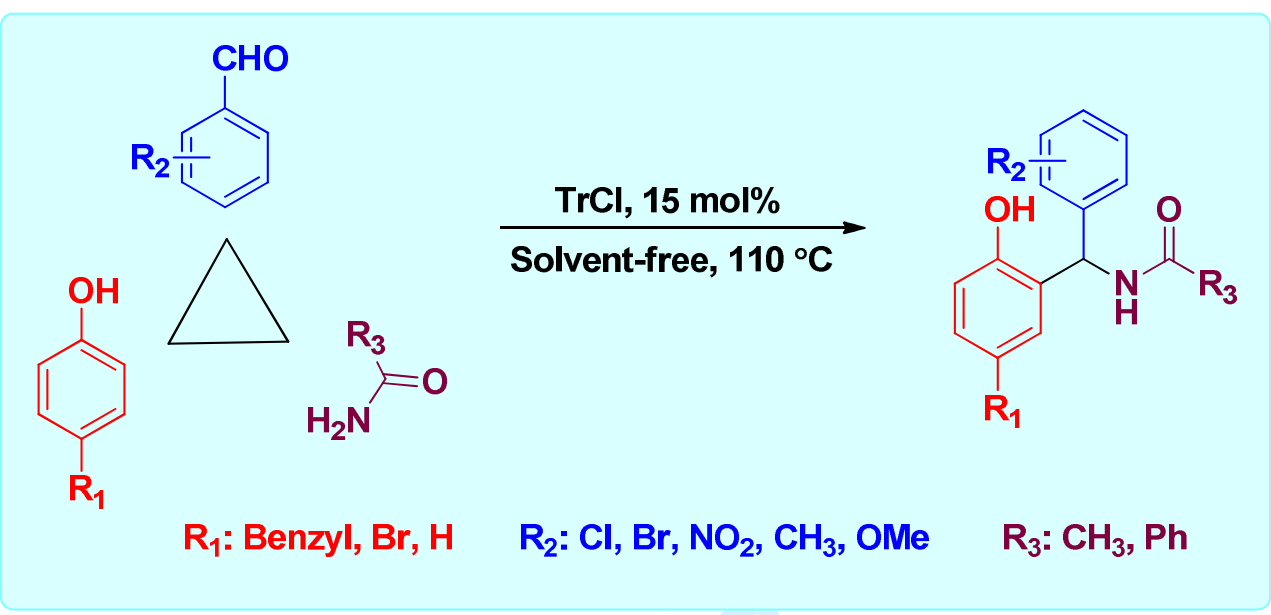




\section{Introduction}

Multi-component reactions (MCRs) achieve important role in combinatorial chemistry due to the ability to preparation of target compounds with more atomic economy and efficiency by the reaction of several compounds together in a single step. Additionally, MCRs increase simplicity and synthetic efficiency on the conventional organic synthesis. ${ }^{1-7}$

Development of organocatalytic transformations in which the reactions are catalyzed by organic molecules has become an area of tremendous importance in current organic synthesis specially from the green chemistry point of view. ${ }^{8}$ Unlike the conventional catalysis, these organocatalysts are advantageous in some ways like high stability, availability of the catalyst, metal reduced toxicity, free nature, and simple reaction conditions and can promote a chemical reaction through various activation modes. 5,9

1-Amidoalkyl-2-naphthols are of importance as they can be easily converted to 1-aminoalkyl-2naphthol derivatives by hydrolysis, which are biologically interesting compounds (Figure 1).

\section{«Figure 1»}

1-Aminoalkyl-2-naphthols have been applied as bradycardiac and hypotensive agents. ${ }^{10,11}$ Also, 1-amidoalkyl-2-naphthols can be converted to 1,3-oxazine derivatives (Figure 1.). ${ }^{12}$

Some pharmaceutical properties have been reported for 1,3-oxazines, such as antibiotic, ${ }^{13}$ antitumor, ${ }^{14}$ and analgesic activities. ${ }^{15}$

Herein, in continuous the previous reports on the synthesis of amido-alkyl-phenols, ${ }^{16-18}$ we have introduced a new protocol for the preparation of amido-alkyl-phenols by the one-pot, multicomponent condensation reaction of various phenols, aromatic aldehydes, and amides using of trityl chloride $(\mathrm{TrCl})$ as a homogeneous organocatalyst under solvent-free and neutral condition (Scheme 1). 


\section{«Scheme 1»}

\section{Results and discussion}

At first, the condensation of 4-benzylphenol $(1 \mathrm{mmol})$ with benzaldehyde $(1 \mathrm{mmol})$ and acetamide $(1.2 \mathrm{mmol})$ was selected as model reaction to optimize the reaction conditions. Then, different conditions such as kind of catalyst, amount of catalyst and temperature, were investigated on the model reaction.

In the next step, to optimize amount of the catalyst and temperature, the model reaction was examined using different amounts of $\mathrm{TrCl}$ at range of $90-130{ }^{\circ} \mathrm{C}$ under solvent-free conditions (Table 1). As Table 1, indicates that, the reaction was efficiently performed in the presence of $15 \mathrm{~mol} \%$ of $\mathrm{TrCl}$ at $110^{\circ} \mathrm{C}$, and afforded the desired product in high yield (Table 1, entry 4).

\section{«Table 1»}

In a proposed mechanism that is supported by the literature, ${ }^{2}$ (Scheme 2), aldehyde was activated by the trityl carbocation. Two resonance forms of activated aldehyde (I and II) could be produced and converted together in a reversible reaction. ${ }^{4,5,19,20,21} \mathbf{I}$ and II were introduced by Oikawa et al as cationic intermediates for the first time. ${ }^{22}$ Therefore, I and $\mathbf{I I}$ act as an activated complex and then react with phenolic compound to give III, which converts to IV by proton transferring. Then, IV could be converted to $\mathbf{V}$ by removing one molecule of triarylmethanol. Afterward, V or IV reacts with amide to obtain VI. Intermediate VI reacts with triarylmethanol to give amido-alkyl-phenols and $\mathrm{TrCl}$.

\section{«Scheme 2»}

To study the efficacy of $\mathrm{TrCl}$ in the synthesis of amido-alkyl-phenols, various aromatic aldehydes containing electron-releasing substituents, electron-withdrawing substituents and halogens were reacted with various phenolic compounds (phenol, 4-benzylphenol and 
4-bromophenol) and amides (acetamide and benzamide) to produce the desired products in moderate to high yields. The results are displayed in Table 2.

«Table 2»

As it is shown in the Scheme 1, acetamido-alkyl-phenols were prepared by the one-pot multicomponent condensation reaction between phenolic compounds, aldehydes and acetamide using $\mathrm{TrCl}$ as catalyst. The mixture was stirred for $180-270 \mathrm{~min}$ at $110^{\circ} \mathrm{C}$ which afforded the desired products in $64-92 \%$. The structures of these mentioned products were confirmed by the IR, $1 \mathrm{H}$ and ${ }^{13} \mathrm{C}$ NMR and mass analysis (Experimental section). In the IR spectra, the absorbance of carbonyl group $(\mathrm{C}=\mathrm{O})$ between $1634-1656 \mathrm{~cm}^{-1}$, two strong peaks at about ca. $3179 \mathrm{~cm}^{-1}$ and $3410 \mathrm{~cm}^{-1}$ related to $v_{\mathrm{O}-\mathrm{H}}$ and $v_{\mathrm{N}-\mathrm{H}}$ stretching vibrations are useful to identify of these compounds. In the ${ }^{1} \mathrm{H}$ NMR spectra of them (for samples in DMSO- $\mathrm{d}_{6}$ solution), the chemical shift of $\mathrm{OH}$ (phenolic hydrogen), $\mathrm{NH}$ (amidic hydrogen) and $\mathrm{CH}_{3}$ (the hydrogens of methyl group) were displayed at about 9.35, 8.5 and 1.82. Also, in ${ }^{13} \mathrm{C}$ NMR spectra (for samples in DMSO- $\mathrm{d}_{6}$ solution), the chemical shift of carbonyl group $(\mathrm{C}=\mathrm{O})$, aliphatic $\mathrm{CH}$ and $\mathrm{CH}_{3}$ were depicted at about 168.4, 56.4 and 22.2, respectively. These important observations helped us to identify the structures of acetamido-alkyl-phenols (Experimental section, 1-9).

In another study, to confirm that in situ generated $\mathrm{HCl}$ or another $\mathrm{H}^{+}$in the reaction condition could not be catalyzed the reaction, the reaction was tested using DABCO $(1,4-$ Diazabicyclo[2.2.2] octane) as a base in which no progress in the reaction was observed. Then, the model reaction was examined in the presence of $\mathrm{TrCl}$ together with $\mathrm{DABCO}$ where in the reaction was successfully carried out and the product was obtained in $75 \%$ within 210 minutes. In these conditions, DABCO as an acid scavenger, absorbs $\mathrm{H}^{+}$and produces $\left[\mathrm{DABCO}-\mathrm{H}_{2}\right] \mathrm{Cl}_{2}$. 
These experiments clearly confirm that in situ prepared $\mathrm{HCl}$ or another source of $\mathrm{H}^{+}$in the reaction conditions; could not be catalyzed the reaction.

In conclusion, we have introduced the one-pot multi-component synthesis of amido-alkylphenols by the reaction of various aromatic aldehydes, phenolic compounds (4-benzylphenol, 4bromophenol and phenol) and amides (acetamide and benzamide) using trityl chloride ( $\mathrm{TrCl})$ as catalyst at $110^{\circ} \mathrm{C}$ under solvent-free conditions.

\section{Experimental}

\section{Materials}

All chemicals were purchased from Fluka or Merck Chemical Companies. Progress of the reactions was monitored by TLC using silica gel SIL G/UV 254 plates. The ${ }^{1} \mathrm{HNMR}(400 \mathrm{MHz})$ and ${ }^{13} \mathrm{CNMR}(100 \mathrm{MHz})$ were recorded on a Bruker AVANCE- DRX. FT-NMR spectrometer $(\delta$ in ppm). Melting points were run on a Büchi B-545 apparatus in open capillary tubes. IR spectra were recorded on a Perkin Elmer PE-1600-FTIR. Mass spectra were run on Agilent Technology (HP) Network Mass Selective Detector 5973 (70eV) apparatus.

\subsection{General procedure for the synthesis of amido-alkyl-phenols using $\operatorname{TrCl}$ :}

To a well-ground mixture of phenolic compound $(1 \mathrm{mmol})$, aldehyde $(1 \mathrm{mmol})$ and amide derivative $(1.2 \mathrm{mmol})$ in a $10 \mathrm{~mL}$ round-bottomed flask connected to a reflux condenser, was added the catalyst $(15 \mathrm{~mol} \%)$, and the resulting mixture was stirred in an oil-bath at $110{ }^{\circ} \mathrm{C}$. After completion of the reaction, as monitored by TLC, the reaction mixture was cooled to room temperature and then petroleum ether $(5 \mathrm{~mL})$ was added to the crude reaction mixture and filtered to separate the catalyst which will be recycled for other reactions. Subsequently, the solid residue (crude product) was purified by plate chromatography on silica gel with n-hexan/ethylacetate (6/4) as an eluent to give the desired amido-alkyl-phenols. 


\subsection{Spectral data of compounds:}

N-((5-benzyl-2-hydroxyphenyl)(phenyl)methyl)acetamide (1): mp: $174-176{ }^{\circ} \mathrm{C} ; \mathrm{IR}\left(\mathrm{KBr}, \mathrm{cm}^{-1}\right)$ : 3410, 3179, 3024, 1644, 1611; ${ }^{1} \mathrm{H}$ NMR (400 MHz, DMSO-d6): $\delta$ (ppm) 1.96 (s, 3H), 3.85 (s, 2H), $6.48(\mathrm{~d}, J=8.4 \mathrm{~Hz}, 1 \mathrm{H}), 6.77(\mathrm{~d}, J=8 \mathrm{~Hz}, 1 \mathrm{H}), 6.99(\mathrm{~d}, J=8 \mathrm{~Hz}, 1 \mathrm{H}), 7.15-7.28(\mathrm{~m}, 6 \mathrm{H})$, 7.37-7.72 (m, 2H), $8.09(\mathrm{~d}, J=8 \mathrm{~Hz}, 2 \mathrm{H}), 8.76\left(\mathrm{~d}, J=8 \mathrm{~Hz}, 1 \mathrm{H}\left(, 9.56(1 \mathrm{H}, \mathrm{s}) \mathrm{ppm} ;{ }^{13} \mathrm{C}\right.\right.$ NMR (100 MHz, DMSO-d $\left.{ }_{6}\right): \delta(p p m) 23.09,40.98,50.61,115.89,121.83,122.14,126.28,127.82$, $128.79,129.26,130.17,132.10,134.41,142.21,145.25,148.17,153.08,169.28 ; \mathrm{MS}: \mathrm{m} / \mathrm{z}=331$ $\left(\mathrm{M}^{+}\right)$.

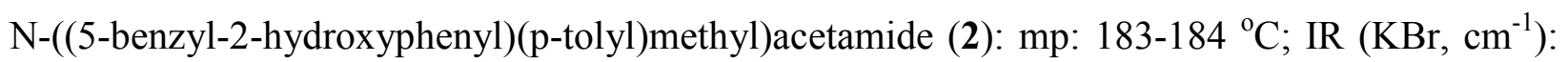
3412, 3246, 3025, 1648, 1610; 1H NMR (400 MHz, DMSO-d $): \delta(p p m) 1.90$ (s, 3H), 2.25 (s, $3 \mathrm{H}), 3.81(\mathrm{~s}, 2 \mathrm{H}), 6.31(\mathrm{~d}, \mathrm{~J}=5.96 \mathrm{~Hz}, 1 \mathrm{H}), 6.71(\mathrm{~d}, J=5.48 \mathrm{~Hz}, 1 \mathrm{H}), 6.87(\mathrm{dd}, J=4.04,1.40$ $\mathrm{Hz}, 1 \mathrm{H}), 7.07-7.28(\mathrm{~m}, 10 \mathrm{H}), 8.47$ (d, $J=5.96 \mathrm{~Hz}, 1 \mathrm{H}), 9.56(\mathrm{~s}, 1 \mathrm{H}) \mathrm{ppm} ;{ }^{13} \mathrm{CNMR}(100 \mathrm{MHz}$; DMSO-d6) $\delta$ (ppm) 21.04, 23.10, 41.03, 50.47, 115.63, 126.23, 127.44, 128.45, 128.53, 128.76, 128.93, 129.01, 129.19, 131.66, 135.96, 140.29, 142.30, 153.04, 168.84; MS: m/z = $345\left(\mathrm{M}^{+}\right)$.

N-((5-benzyl-2-hydroxyphenyl)(3-nitrophenyl)methyl)acetamide (3): mp: 210-215 ${ }^{\circ} \mathrm{C}$; IR (KBr, cm-1): 3381, 3025, 2733,1635, 1606; ${ }^{1} \mathrm{H}$ NMR (400 MHz, DMSO-d $\left.\mathrm{d}_{6}\right): \delta(\mathrm{ppm}) 1.88(\mathrm{~s}, 3 \mathrm{H})$, $3.79(\mathrm{~s}, 2 \mathrm{H}), 6.44(\mathrm{~d}, J=7.6 \mathrm{~Hz}, 1 \mathrm{H}), 6.74(\mathrm{~d}, J=8 \mathrm{~Hz}, 1 \mathrm{H}), 6.81(\mathrm{~s}, 1 \mathrm{H}), 6.92(\mathrm{~d}, J=8 \mathrm{~Hz}, 1 \mathrm{H})$, 7.13-7.58 (m, 8H), $7.59\left(\mathrm{~d}, J=4.8 \mathrm{~Hz}, 1 \mathrm{H}\left(, 8.41(1 \mathrm{H}, \mathrm{s}), 9.39(1 \mathrm{H}, \mathrm{s}) \mathrm{ppm} ;{ }^{13} \mathrm{CNMR}(100 \mathrm{MHz}\right.\right.$; DMSO-d $\left._{6}\right) \delta$ (ppm) 22.89, 40.97, 51.48, 115.58, 124.14, 126.26, 127.38, 127.83, 128.76, 128.82, $128.93,128.98,129.25,129.58131 .25,133.13,141.85,142.15,153.72,168.78 ; \mathrm{MS}: \mathrm{m} / \mathrm{z}=376$ $\left(\mathrm{M}^{+}\right)$.

N-((5-benzyl-2-hydroxyphenyl)(2-nitrophenyl)methyl)acetamide (4): mp: 226-228 ${ }^{\circ} \mathrm{C}$; IR (KBr, cm-1): 3403, 3271, 3027, 1656, 1614; ${ }^{1} \mathrm{H}$ NMR (400 MHz, DMSO-d 6 ): $\delta$ (ppm) 1.79 (s, 3H), 
3.68(s, 2H), 6.60-6.70 (m, 3H), $6.82(\mathrm{dd}, J=6.24,1.96 \mathrm{~Hz}, 1 \mathrm{H}), 7.01-7.18(\mathrm{~m}, 3 \mathrm{H}), 7.32-7.34$ (m, 2H), 7.34-7.41 (m, 1H), $7.75(\mathrm{~s}, 1 \mathrm{H}), 7.77(\mathrm{~s}, 1 \mathrm{H}), 7.78(\mathrm{~d}, J=7.36 \mathrm{~Hz}, 1 \mathrm{H}), 8.43(\mathrm{~d}, J=$ $7.96 \mathrm{~Hz}, 1 \mathrm{H}), 9.36$ (s, 1H) ppm; ${ }^{13} \mathrm{CNMR}(100 \mathrm{MHz}$; DMSO-d 6 ) $\delta(\mathrm{ppm}) 22.28,46.91,56.00$, $115.01,124.06,125.73,126.80,127.65,128.10,128.43,128.48,128.63,129.54,130.89,132.63$, 135.98, 141.53, 148.81, 153.00, 168.41; MS: $\mathrm{m} / \mathrm{z}=376\left(\mathrm{M}^{+}\right)$.

N-((5-benzyl-2-hydroxyphenyl)(phenyl)methyl)benzamide (5): mp: 204-206 ${ }^{\circ} \mathrm{C}$; IR $\left(\mathrm{KBr}, \mathrm{cm}^{-1}\right)$ : 3403, 3062, 3028, 1630, 1603; 1H NMR (400 MHz, DMSO-d 6 ): $\delta$ (ppm) 3.78 (s, 2H), 6.46 (d, $J$ $=8.4 \mathrm{~Hz}, 1 \mathrm{H}), 6.67-6.92(\mathrm{~m}, 11 \mathrm{H}), 7.13-7.27(\mathrm{~m}, 7 \mathrm{H}), 8.23(\mathrm{~d}, J=8.4 \mathrm{~Hz}, 1 \mathrm{H}), 9.25(\mathrm{~s}, 1 \mathrm{H})$ ppm; ${ }^{13} \mathrm{CNMR}\left(100 \mathrm{MHz}\right.$; DMSO-d $\left.\mathrm{d}_{6}\right) \delta$ (ppm) 41.04, 56.45, 111.91, 112.47, 115.41, 115.55, $126.21,128.44,128.56,128.74,128.87,128.93,131.14,132.07,142.39,151.31,153.50,153.58$, 168.59; MS: $\mathrm{m} / \mathrm{z}=393\left(\mathrm{M}^{+}\right)$.

N-((5-bromo-2-hydroxyphenyl)(4-chlorophenyl)methyl)acetamide (6): mp: 220-225 ${ }^{\circ} \mathrm{C}$; IR (KBr, cm-1): 3309, 3074, 2980, 2739, 1649, 1631; ${ }^{1} \mathrm{H}$ NMR (400 MHz, DMSO-d 6 ): $\delta$ (ppm) $1.85(\mathrm{~s}, 3 \mathrm{H}), 5.95(\mathrm{~d}, J=8.4 \mathrm{~Hz}, 1 \mathrm{H}), 6.68(\mathrm{~s}, 2 \mathrm{H}), 6.99(\mathrm{~d}, J=8 \mathrm{~Hz}, 2 \mathrm{H}), 7.23-7.35(\mathrm{~m}, 3 \mathrm{H})$ 8.66 (s, 1H), 9.35 (s, 1H) ppm; ${ }^{13} \mathrm{CNMR}(100 \mathrm{MHz}$; DMSO-d6) $\delta$ (ppm) 24.19, 56.36, 116.70, $129.78,130.14,130.50,132.84,133.97,143.90,158.02,169.99 ; \mathrm{MS}: \mathrm{m} / \mathrm{z}=354\left(\mathrm{M}^{+}\right)$.

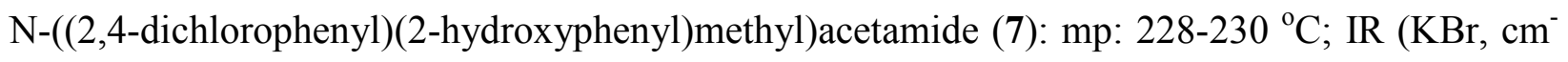
$\left.{ }^{1}\right): 3300.7,3065,2808,1634,1613 ;{ }^{1} \mathrm{H}$ NMR (400 MHz, DMSO-d 6 ): $\delta$ (ppm) 1.85 (s, 3H), 6.33 $(\mathrm{d}, J=8 \mathrm{~Hz}, 1 \mathrm{H}), 6.47(\mathrm{~d}, J=8 \mathrm{~Hz}, 1 \mathrm{H}), 6.71(\mathrm{~d}, J=8 \mathrm{~Hz}, 1 \mathrm{H}), 6.91(\mathrm{~d}, J=7.6 \mathrm{~Hz}, 1 \mathrm{H}), 7.14-$ $7.81(\mathrm{~m}, J=8 \mathrm{~Hz}, 4 \mathrm{H}), 7.96(\mathrm{~s}, 1 \mathrm{H}), 8.69(\mathrm{~s}, 1 \mathrm{H}) \mathrm{ppm} ;{ }^{13} \mathrm{CNMR}(100 \mathrm{MHz}$; DMSO-d 6 ) $\delta(\mathrm{ppm})$ $24.08,58.14,117.91,124.91,125.23,127.41,129.90,130.08,130.48,133.03,133.9,134.12$, 143.26, 154.17, 170.50; MS: $\mathrm{m} / \mathrm{z}=310\left(\mathrm{M}^{+}\right)$. 
N-((5-bromo-2-hydroxyphenyl)(2-bromophenyl)methyl)acetamide (8): mp: $197-200 \quad{ }^{\circ} \mathrm{C}$; IR $\left(\mathrm{KBr}, \mathrm{cm}^{-1}\right): 3300,3064,2808,1634,1613 ;{ }^{1} \mathrm{H}$ NMR (400 MHz, DMSO-d 6 ): $\delta$ (ppm) $1.21(\mathrm{~s}$, 3H), $6.45(\mathrm{~s}, 1 \mathrm{H}), 6.61(\mathrm{~s}, 1 \mathrm{H}), 6.98(\mathrm{~s}, 1 \mathrm{H}), 7.06-7.32(\mathrm{~m}, 5 \mathrm{H}), 7.55(\mathrm{~d}, \mathrm{~J}=6.8 \mathrm{~Hz}, 1 \mathrm{H}), 8.69(\mathrm{~s}$, 1H) ppm; ${ }^{13} \mathrm{CNMR}\left(100 \mathrm{MHz} ; \mathrm{DMSO}-\mathrm{d}_{6}\right) \delta(\mathrm{ppm}) 22.82,51.72,126.26,127.17,127.86,128.09$, $128.67,128.86,129.10,129.34,133.25,141.20,141.42,169.66 ; \mathrm{MS}: \mathrm{m} / \mathrm{z}=399\left(\mathrm{M}^{+}\right)$.

N-((5-bromo-2-hydroxyphenyl)(2,5-dimethoxyphenyl)methyl)acetamide (9): mp: $215-217^{\circ} \mathrm{C}$; IR $\left(\mathrm{KBr}, \mathrm{cm}^{-1}\right): 3372,3267,2854,1652 ; 1 \mathrm{H} \mathrm{NMR}\left(400 \mathrm{MHz}, \mathrm{DMSO}-\mathrm{d}_{6}\right): \delta(\mathrm{ppm}) 1.82(\mathrm{~s}, 3 \mathrm{H})$, $3.31(\mathrm{~s}, 3 \mathrm{H}), 3.76(\mathrm{~s}, 3 \mathrm{H}), 6.30(\mathrm{~d}, J=6 \mathrm{~Hz}, 1 \mathrm{H}), 6.69(\mathrm{~d}, J=8.4 \mathrm{~Hz}, 1 \mathrm{H}), 6.87(\mathrm{~d}, J=6.4 \mathrm{~Hz}$, 1H), 7.09-7.28 (m, 4H), $8.50(\mathrm{~d}, J=9.2 \mathrm{~Hz}, 1 \mathrm{H}), 9.35$ (s, 1H) ppm; ${ }^{13} \mathrm{CNMR}(100 \mathrm{MHz}$; DMSO$\left.\mathrm{d}_{6}\right) \delta(\mathrm{ppm}) 23.12,41.05,50.46,115.68,126.26,127.32,128.33,128.48,128.79,128.96,129.05$, 131.78, 141.55, 142.32, 152.94, 168.75; MS: $\mathrm{m} / \mathrm{z}=380\left(\mathrm{M}^{+}\right)$.

\section{Acknowledgements}

The authors gratefully acknowledge partial support of this work by the Research Affairs Office of Bu-Ali Sina University (Grant number 32-1716 entitled development of chemical methods, reagents and molecules), and Center of Excellence in Development of Chemical Method (CEDCM), Hamedan, I.R. Iran.

\section{Reference:}

[1] Zhu, J.; Bienayme, H.; Multicomponent Reactions, Eds., Wiley, Weinheim, 2005.

[2] Khazaei, A.; Zolfigol, M. A.; Moosavi-Zare, A. R.; Abi, F.; Zare, A.; Kaveh, H.; Khakyzadeh, V.; Kazem-Rostami, M.; Parhami A.; Torabi-Monfared, H.; Tetrahedron, 2013, 69, 212. 
[3] Zolfigol, M. A.; Khazaei, A.; Moosavi-Zare, A. R.; Zare A.; Khakyzadeh, V.; Appl. Catal AGen. 2011, 400, 70.

[4] Khazaei, A.; Zolfigol, M. A.; Moosavi-Zare, A. R.; Zare, A.; Parhami A.; Khalafi-Nezhad, A.; Appl. Catal. A: Gen. 2010, 386, 179.

[5] Khazaei, A.; Zolfigol, M. A.; Moosavi-Zare, A. R.; Zare, A.; Khojasteh, M.; Asgari, Z.; Khakyzadeh V.; Khalafi-Nezhad, A.; Catal. Commun. 2012, 20, 54.

[6] Zolfigol, M. A.; Khakyzadeh, V.; Moosavi-Zare, A. R.; Zare, A.; Azimi, S. B.; Asgari Z.; Hasaninejad, A.; C. R. Chim. 2012, 15, 719.

[7] Moosavi-Zare, A. R.; Zolfigol, M. A.; Daraei, M.; Synlett, 2014, 25, 1173.

[8] Dondoni, A.; Massi, A.; Angew. Chem. Int. Ed. 2008, 47, 4638.

[9] Verma, S.; Jain, L.S.; Sain, B.; Tetrahedron Lett. 2010, 51, 6897.

[10] Shaterian, H. R.; Yarahmadi, H.; Ghashang, M.; Bioorg. Med. Chem. Lett. 2008, 18, 788.

[11] Shen, A.Y.; Tsai C. T.; Chen, C. L.; Eur. J. Med. Chem. 1999, 34, 877.

[12] Damodiran, M.; Selvam N. P.; Perumal, P. T.; Tetrahedron Lett. 2009, 50, 5474.

[13] Kusakabe, Y.; Nagatsu, J.; Shibuya, M.; Kawaguchi, O.; Hirose C.; Shirato, S.; J. Antibiot. 1972, 25,44 .

[14] Renullard, S.; Rebhun, L. I.; Havic G. A.; Kupchan, S. M.; Science, 1975, 189, 1002.

[15] Lesher, G. Y.; Surrey, A. R.; J. Am. Chem. Soc. 1955, 77, 636.

[16] Selvam, N. P.; Perumal P. T.; Tetrahedron, 2008, 64, 2972.

[17] Damodiran, M.; Selvam, N. P.; Perumal P. T.; Tetrahedron Lett. 2009, 50, 5474.

[18] Das, B.; Laxminarayana, K.; Thirupathi, P.; Ramarao B.; Synlett, 2007, 20, 3103. 
[19] Khalafi-Nezhad, A.; Parhami, A.; Zare, A.; Moosavi-Zare, A. R.; Hasaninejad, A.; Panahi, F.; Synthesis, 2008, 617.

[20] Khalafi-Nezhad, A.; Parhami, A.; Zare, A.; Nasrollahi Shirazi, A.; Moosavi-Zare, A. R.; Hasaninejad, A.; Can. J. Chem. 2008, 86, 456.

[21] Zare, A.; Merajoddin, M.; Moosavi-Zare, A. R.; Zarei, M.; Chin. J. Catal. 2014, 35, 85.

[22] Oikawa, M.; Yoshizaki, H.; Kusumoto, S.; Synlett, 1998, 757. 


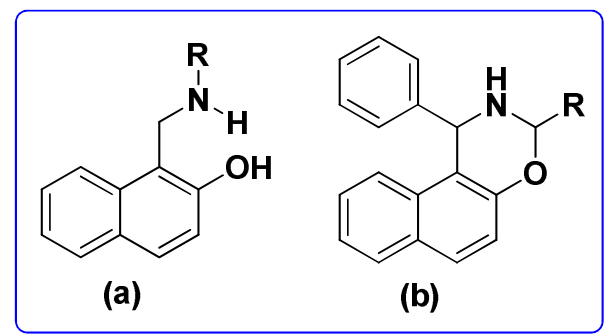

Figure 1. The general structures of 1-aminoalkyl-2-naphthols (a) and 1,3- oxazines (b).

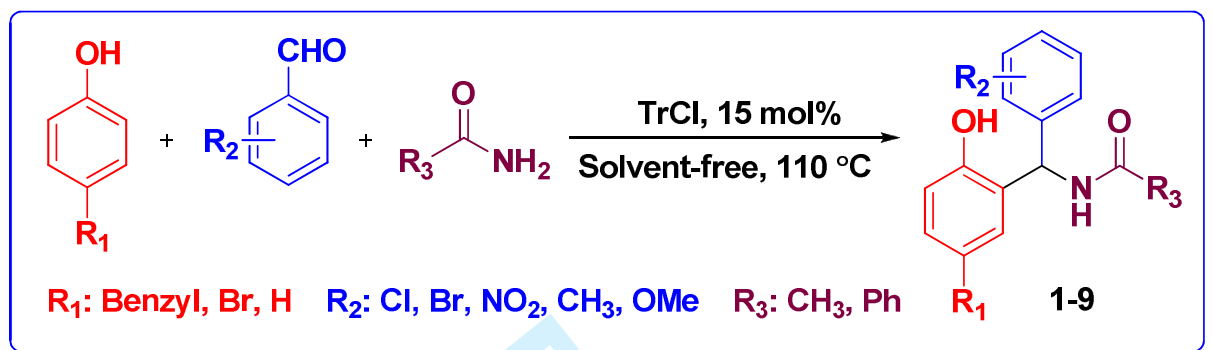

Scheme 1. The Synthesis and general structure of amido-alkyl-phenols. 


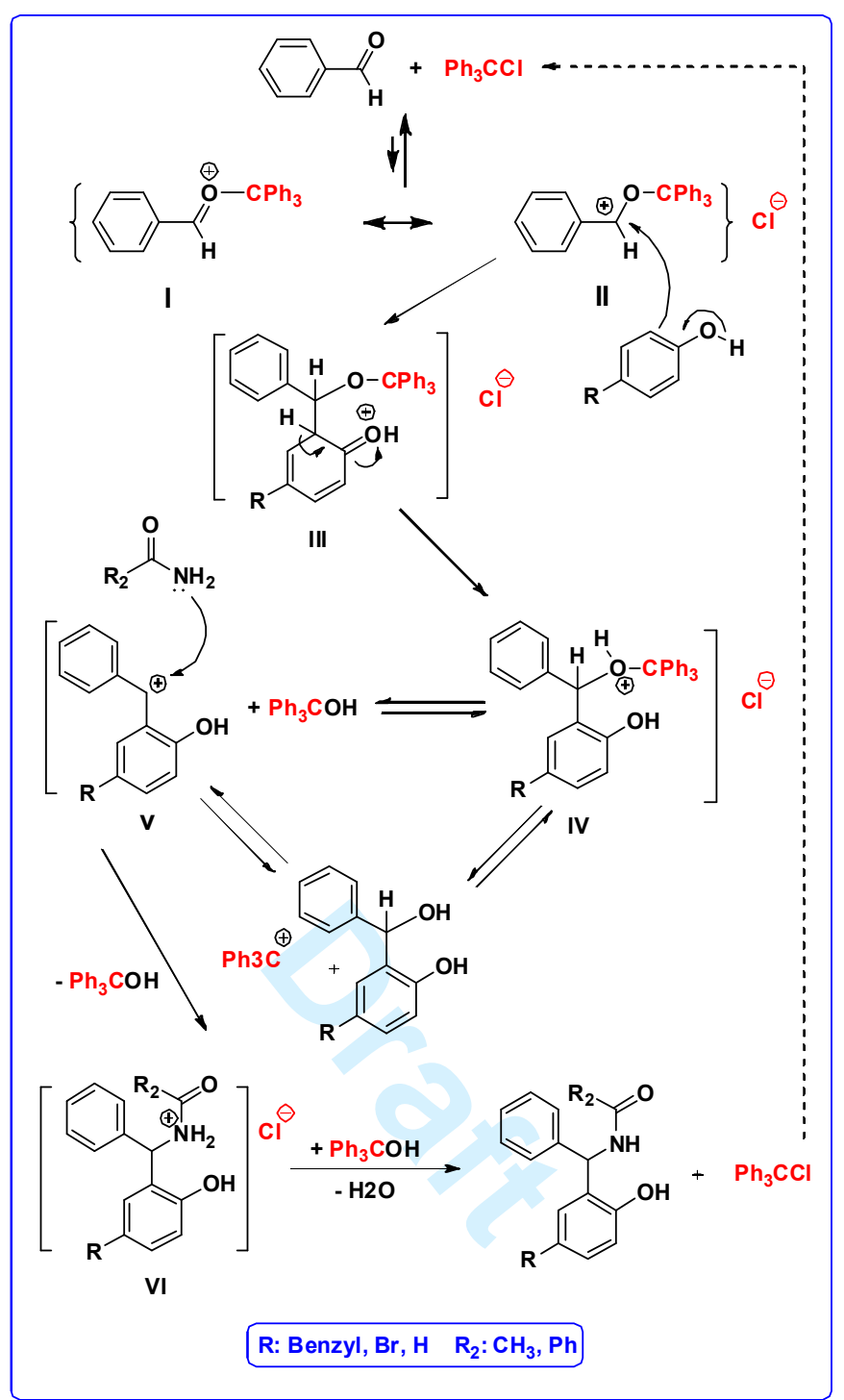

Scheme 2. The proposed mechanism for the synthesis of amido-alkyl-phenols. 
Table 1. Effect of different amounts of the catalyst and temperature on the condensation of 4benzylphenol $(1 \mathrm{mmol})$ with benzaldehyde $(1 \mathrm{mmol})$ and acetamide $(1.2 \mathrm{mmol})$ under solventfree conditions.

\begin{tabular}{ccccc}
\hline Entry & $\begin{array}{c}\text { Mol\% of } \\
\text { Catalyst }\end{array}$ & Temp. $\left({ }^{\circ} \mathrm{C}\right)$ & Time (min) & Yield $^{\mathrm{a}}(\%)$ \\
\hline 1 & - & 110 & 240 & - \\
2 & 5 & 110 & 300 & 55 \\
3 & 10 & 110 & 270 & 60 \\
4 & 15 & 110 & 210 & 75 \\
5 & 15 & 90 & 270 & 69 \\
6 & 15 & 130 & 210 & 75 \\
7 & 20 & 110 & 210 & 75 \\
\hline
\end{tabular}

${ }^{\mathrm{a}}$ Isolated yield. 
Table 2. The synthesis of amido-alkyl-phenols using $\mathrm{TrCl}$ at $110{ }^{\circ} \mathrm{C}$ under solvent-free conditions.

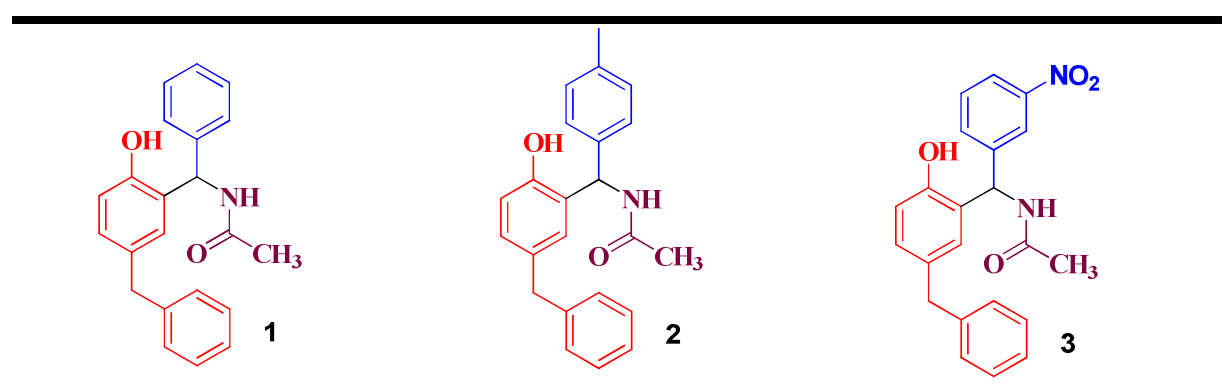

[Time: $210 \mathrm{~min} /$ Yield: $75 \%$ ] Mp: $174-175^{\circ} \mathrm{C}\left(174-176^{\circ} \mathrm{C}\right)^{7}$

[Time: 180 min / Yield: $64 \%$ ] Mp: $182-185^{\circ} \mathrm{C}\left(183-184^{\circ} \mathrm{C}\right)^{7} \quad \mathrm{Mp}: 210-212^{\circ} \mathrm{C}\left(210-215^{\circ} \mathrm{C}\right)^{7}$<smiles>CC(=O)NC(c1ccccc1)c1ccccc1O</smiles>

[Time: $180 \mathrm{~min} /$ Yield: $87 \%$ ] Mp: $226-228^{\circ} \mathrm{C}\left(226-228^{\circ} \mathrm{C}\right)^{7}$

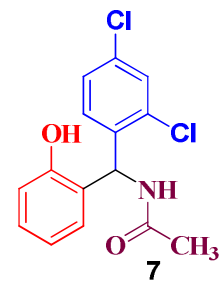

[Time: $270 \mathrm{~min} /$ Yield: $92 \%$ ] Mp: $227-230^{\circ} \mathrm{C}\left(228-230^{\circ} \mathrm{C}\right)^{7}$

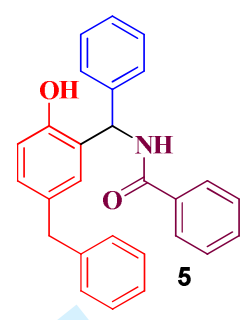

[Time: $240 \mathrm{~min} /$ Yield: $79 \%$ ] Mp: $202-204^{\circ} \mathrm{C}\left(204-206^{\circ} \mathrm{C}\right)^{7}$

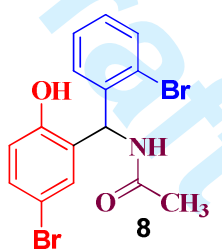

[Time: $210 \mathrm{~min} /$ Yield: $78 \%$ ] Mp: $197-199^{\circ} \mathrm{C}\left(197-200^{\circ} \mathrm{C}\right)^{7}$

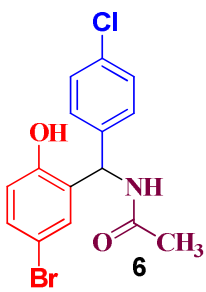

[Time: $210 \mathrm{~min} /$ Yield: $87 \%$ ] Mp: $222-225^{\circ} \mathrm{C}\left(220-225^{\circ} \mathrm{C}\right)^{7}$<smiles>COc1ccc(OC)c(C(NC(C)=O)c2cc(Br)ccc2O)c1</smiles>

[Time: $280 \min /$ Yield: $75 \%$ ] Mp: $214-217^{\circ} \mathrm{C}\left(215-217^{\circ} \mathrm{C}\right)^{7}$

${ }^{\text {a }}$ Isolated yield. 